\title{
ROC Curve in Evaluation of Diagnostic Value of Serum 25-hydroxyvitamin-D for PCOS with Depression
}

\author{
Ying Liu and Qianhui Qi \\ Department of Gynaecology, The Second Affiliated Hospital of Guangzhou Medical University, Guangdong Province, China
}

\begin{abstract}
The objective of this study was to apply receiver operating characteristic (ROC) curve to evaluate diagnostic value of serum 25hydroxyvitamin-D [25-(OH)D] for polycystic ovarian syndrome (PCOS) combined with depression. Serum 25-(OH)D level was determined in 106 PCOS patients. Beck depression scale (BDI) and ROC curve were used to assess the objective. Results showed that there were 40 cases $(37.74 \%)$ in combined depression group, and $66(62.26 \%)$ in non-combined depression group. Number of patients with depression family history and BDI score in combined depression group were higher than those in non-combined depression group ( $p=0.002$ and $p<0.001$, respectively). Serum $25-(\mathrm{OH}) \mathrm{D}$ level in combined depression group was lower than that in non-combined depression group $(p<0.001)$. Area under ROC curve for prediction of PCOS combined with depression by serum $25-(\mathrm{OH}) \mathrm{D}$ was $0.829(95 \% \mathrm{Cl}=0.746-0.911)$. Clinical point value of diagnosis of serum $25-(\mathrm{OH}) \mathrm{D}$ was $10.89 \mu \mathrm{g} / \mathrm{L}$. Sensitivity was $75 \%$ and specificity was $78.8 \%$.
\end{abstract}

Key Words: Serum, 25-hydroxyvitamin-D [25-(OH)D], Polycystic ovarian syndrome (PCOS) , Depression, Receiver operating characteristic (ROC) curve.

How to cite this article: Liu Y, Qi Q. ROC Curve in Evaluation of Diagnostic Value of Serum 25-hydroxyvitamin-D for PCOS with Depression. J Coll Physicians Surg Pak 2020; 30(06):659-661 https://doi.org/10.29271/jcpsp.2020.06.659.

Polycystic ovarian syndrome (PCOS) is a special disease in which abnormal glucose metabolism and reproductive dysfunction coexist. ${ }^{1}$ PCOS has the features of high morbidity, complicated conditions of disease, and unclear pathogenesis. The clinical manifestations of PCOS mainly include oligomenorrhea, amenorrhea, crinosity, acne, alopecia seborrhoeica, ovarian morphologic change and abnormal sex hormone level. Depression is a common complication of PCOS patients. One study reported that depression could influence body immune system and thus cause immune activation. ${ }^{2}$ Vitamin $D$ is a fat-soluble steroid derivative, and it is also one of the main hormones to maintain calcium and phosphorus metabolic balance. One study showed that Vitamin D deficiency could play an important role in the occurrence and development of depression. ${ }^{3}$ At present, there are still a few studies about the application of Vitamin D level in the diagnosis of PCOS combined with depression. The $25-(\mathrm{OH}) \mathrm{D}$ is main existence form of Vitamin $\mathrm{D}$ in the body. Currently, there is still lack of knowledge about the diagnostic clinical point of 25-(OH)D for PCOS combined with depression.

Correspondence to: Ying Liu, Department of Gynaecology, The Second Affiliated Hospital of Guangzhou Medical University, Guangdong Province, China

E-mail:nlt4km@163.com

Received: October 24, 2019; Revised: December 20, 2019;

Accepted: December 23, 2019

DOI: https://doi.org/10.29271/jcpsp.2020.06.659
The objective of this study was to apply ROC curve to evaluate the diagnostic value of serum 25-(OH)D for PCOS combined with depression and the possible diagnostic critical point.

This study was conducted at the Second Affiliated Hospital of Guangzhou Medical University, China, from September 2017 to August 2019, and approved by the Medical Ethics Committee. One hundred and six PCOS patients were chosen at random for this study. The inclusion criterion were that patients complied with PCOS diagnostic criteria, revised by American Society for Reproductive Medicine and European Society of Human Reproduction and Embryology, patients did not take Vitamin D and antidepressant agents within three months before the diagnosis. Exclusion criteria were that patients combined with ovarian generating androgen or adrenal tumors, and patients combined with Cushing's syndrome, congenital adrenal cortex hyperplasia, hypothyroidism, and hyperprolactinemia, and other patients causing androgen excess and anovulation; patients with the history of euglycemic agent, lipid-lowering and hormonotherapy within 3 months; patients combined with serious physical diseases.

Beck depression scale (BDI) was used to assess whether PCOS patients have depression and if so, the depression degree. BDI score 14-19 was mild depression, 20-28 was moderate depression, and 29-63 was severe depression. Based on the assessment result, 106 patients were classified into combined depression group and non-combined depression group. Depression family history situations of patients were collected. Venous blood was collected on empty stomach for all patients on the 
$3^{\text {rd }}-5^{\text {th }}$ days of menstrual cycle $\square$ this period of time belongs to the early follicular period, which can reflect the functional state of the ovary). No date limit was for those with amenorrhea. The serum wascentrifugallyseparated. Theenzyme linked immunosorbent assay (ELISA) was used to test serum 25-(OH)D level. Data was analysed with SPSS version 25.0 software. The measurement data were expressed with mean \pm SD and tested with independent-sample t-test. The enumeration data were expressed with $n(\%)$ and tested with Chi-square test. ROC curve was used to assess the sensitivity and specificity of 25$(\mathrm{OH}) \mathrm{D}$ in the prediction of PCOS combined with depression. Statistical significance was defined as a $p$-value of $<0.05$.

The age of $106 \mathrm{PCOS}$ patients ranged from 21 to 30 years ( 25.17 $\pm 2.85)$, and 23 cases $(21.70 \%)$ had the depression family history. There were 40 cases (37.74\%) in combined depression group, and 66 cases (62.26\%) in non-combined depression group. The number of patients with depression family history in combined depression group was 15 cases (37.50\%), higher than that in non-combined depression group 8 cases (12.12\%) $(p=0.002)$. BDI score of patients in combined depression group was $23.11 \pm 1.46$, higher than that in non-combined depression group $(6.03 \pm 0.60, p<0.001)$.

Serum 25-(OH)D of patients in combined depression group was $(10.92 \pm 5.85) \mu \mathrm{g} / \mathrm{L}$, lower than that in non-combined depression group $(21.92 \pm 8.07) \mu \mathrm{g} / \mathrm{L}(\mathrm{p}<0.001)$. The area under the ROC curve for prediction of PCOS combined with depression by serum $25-(\mathrm{OH}) \mathrm{D}$ was $0.829(95 \% \mathrm{Cl}=0.746-0.911)$. When 25 $(\mathrm{OH}) \mathrm{D} \leq 10.89 \mu \mathrm{g} / \mathrm{L}$, the maximum sensitivity $(75 \%)$ and specificity (78.8\%) appeared, as shown in Figure 1.

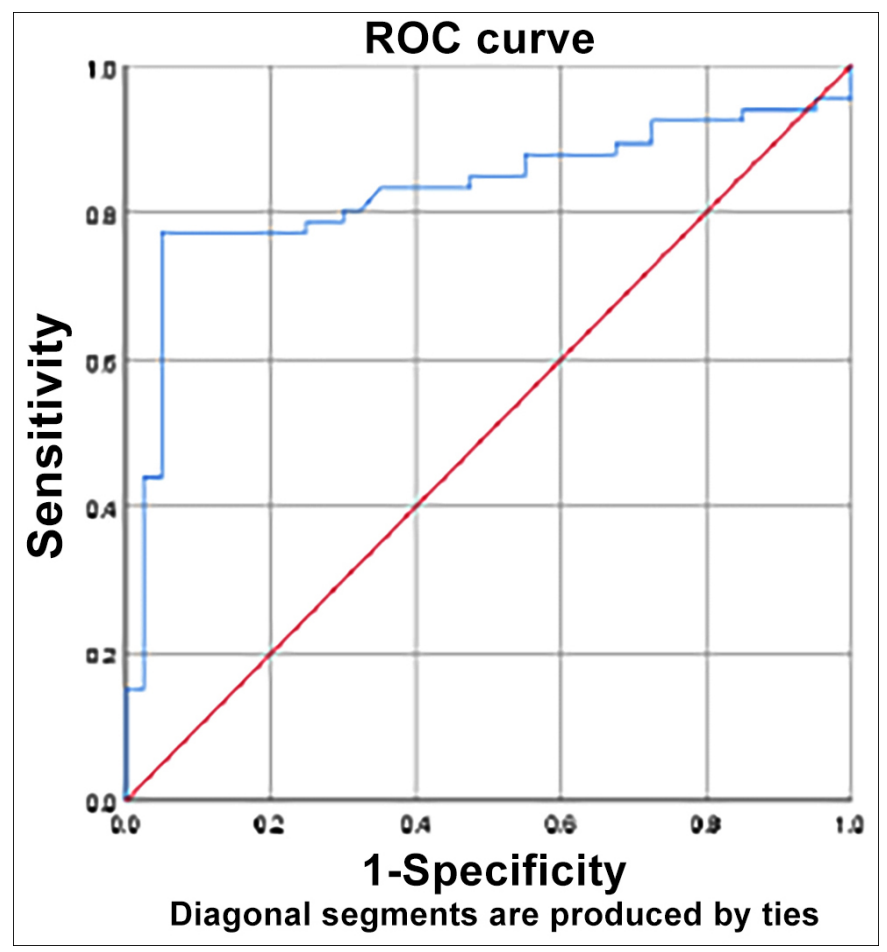

Figure1: Sensitivity and specificity of ROC curve in the assessment of prediction of PCOS combined with depression by serum 25-(OH)D.
This study found that the number of patients with depression family history and BDI score in non-combined depression group werelowerthan those in combined depression group. It is, therefore, inferred that depression family history might be related to PCOS combined with depression. Serum $25-(\mathrm{OH}) \mathrm{D}$ is a Vitamin $D$ derivative with the highest concentration in the circulation. With the long half-life period, it is free from the influence of calcium intake. Hence, it is a common clinical index to judge Vitamin D level in the body. ${ }^{4}$ This study showed that serum 25 $(\mathrm{OH}) \mathrm{D}$ level of PCOS patients combined with depression was lower than that of PCOS patients without depression, indicating that the decline of serum 25-(OH)D level plays an important role in the occurrence and development of PCOS patients combined with depression. This conclusion was basically consistent with the result of Moran etal. ${ }^{5}$

The area under the ROC curve of serum $25-(\mathrm{OH}) \mathrm{D}$ was 0.829 , indicating that serum $25-(\mathrm{OH}) \mathrm{D} \leq 10.89 \mu \mathrm{g} / \mathrm{L}$ as a diagnostic clinical point of PCOS combined with depression was accurate, with high sensitivity and specificity. Besides, the positive and negative predictive values are large. It has good authenticity as the diagnostic test.

At present, there is still no effective treatment method for PCOS. The common therapeutic methods include ovulation induction, antiandrogen and enhancement of insulin sensitivity, etc. It was reported that supplementing Vitamin D could effectively improve depressive symptoms. ${ }^{6}$ However, there is still no study about whether supplementing Vitamin D can improve depression of PCOS patients, so further research in this aspect is required.

In conclusion, serum 25-(OH)D has certain value in the diagnosis of PCOS combined with depression. However, since the sample size in this study is relatively small, large-scale, multi-centre and prospective clinical research is expected to verify the conclusion.

\section{ETHICALAPPROVAL:}

Ethical approval from the Ethics Committee of the Second Affiliated Hospital of Guangzhou Medical University was obtained prior to initiation of the sutdy.

\section{PATIENTS' CONSENT:}

Informed consents have been obtained from all patients to publish the data concerning this case.

\section{CONFLICT OF INTEREST:}

Authors declared no conflict of interest.

\section{AUTHORS' CONTRIBUTION:}

YL: Drafting the work; the acquisition, analysis and interpretation of data for the work, final approval of the version to be published.

QQ: Revising it critically for important intellectual content.

\section{REFERENCES}

1. Delitala AP, Capobianco G, Delitala G, Cherchi PL, Dessole S. Polycystic ovary syndrome adipose tissue and metabolic 
syndrome. Arch Gynecol Obstet 2017; 296(3):405-19.

2. Eyre H, Baune BT. Neuroplastic changes in depression: A role for the immune system. Psychoneuroendocrinology 2012; 37(9):1397-416.

3. Schaad KA, Bukhari AS, Brooks DI, Kocher JD, Barringer ND. The relationship between vitamin $\mathrm{D}$ status and depression in a tactical athlete population. J Int Soc Sports Nutr 2019; 16(1):40.

4. Holick MF, Binkley NC, Bischoff-Ferrari HA, Gordon CM,
Hanley DA, Heaney RP, et al. Evaluation treatment and prevention of vitamin $D$ deficiency: An endocrine society clinical practice guideline. J Clin Endocrinol Metab 2011; 96(7):1911-30.

5. Moran LJ, Teede HJ, Vincent AJ. Vitamin D is independently associated with depression in overweight women with and without PCOS. Gynecol Endocrinol 2015; 31(3):179-82.

6. Bertone Johnson ER. Vitamin $D$ and the occurrence of depression: Causal association or circumstantial evidence? Nutrition Reviews 2010; 67(8):481-92. 